\title{
Problemas actuales de los currículos en la educación física en Iberoamérica. Una revisión sistemática
}

\author{
Current problems of curricula in physical education in Latin America. \\ A systematic review \\ *MC. Abilio Coutín, *Dr. Gabriel Gastélum, *Dr. Julio César Guedea
}

Coutín, A., Gástelum, G. \& Guedea, JC. (2018). Problemas actuales de los currículos en la educación física en Iberoamérica. Una revisión sistemática. Revista Ciencias de la Actividad Física UCM, N²19(2) julio-diciembre, 1-9.

DOI: http://doi.org/10.29035/rcaf.19.2.1

\begin{abstract}
RESUMEN
El estudio del currículo ha llamado la atención no sólo de docentes y expertos en pedagogía, sino de toda la sociedad educativa, ante la búsqueda de respuestas o líneas de acción para mejorar la educación actual. La intención del presente trabajo es introducir al lector a las implicaciones teóricas y prácticas que confiere el término currículo de la educación física (EF). Objetivo: Analizar las problemáticas sobre los currículos actuales de la EF en Iberoamérica. Método: se utilizó una revisión sistemática, de artículos que evaluaron los diseños curriculares y su problemática en diversos países, publicados desde 2013 hasta la actualidad. La búsqueda de artículos se realizó en las bases de datos "Scielo", "Scopus", "Web of Science," "Redalyc" y Google académico. Utilizando los siguientes descriptores, Curricular, Educación Física, Iberoamérica. En términos de búsqueda bibliográfica en los idiomas portugués/ inglés/español. Resultados: de 35 artículos analizados, sólo 6 artículos se vincularon con los descriptores. Conclusión: Se denuncia esa poca claridad conceptual, ese ensayo error; esa falta de sustento científico, esa incongruencia que ha existido en los distintos intentos por llevar a cabo diversas acepciones curriculares, pero también que sigue siendo una asignatura de interés para los estudiantes.
\end{abstract}

\section{PALABRAS CLAVE}

Educación Física, Curricular, Iberoamérica.

\begin{abstract}
Curriculum studies has kept the attention not only from pedagogues and teachers, but from the entire educational society, looking for answers and lines of action for the improvement of current education. The purpose of this study is to introduce to the audience the theoretical and practical implications that are involved within the term curriculum in Physical Education (P.E). Objective: To analyze the current curriculum problematics in P.E across Latin America. Methodology: A systematic review of different peer-reviewed articles was made, articles that evaluated curriculum design and their problematics within different countries, articles published from 2013 to present. Research engines used were data bases such as "Scielo", "Scopus", "Web of Science," "Redalyc" and Google scholar, utilizing descriptors, Curriculum, Physical Education, and Latin America. Languages of articles were selected from Portuguese, English and Spanish. Results: From a total of 35 articles, only 6 were selected according to descriptors such as Curriculum, Physical Education, Latin America. Conclusion: It can be said there is a lack conceptual clarity, lack of scientific rigor, existing incongruence in diverse attempts to involve different curriculum meanings, but it seems to be a subject were students are still showing interest.
\end{abstract}

\section{Key words}

Physical Education, Curriculum, Latin America.

* Facultad de Ciencias de la Cultura Física, Universidad Autónoma de Chihuahua, México. 


\section{INTRODUCCIÓN}

La EF se ha mantenido estática en el tiempo, su involución ha sido motivo de preocupación para los grandes investigadores de esta disciplina, quienes mencionan que se ha ralentizado en el transcurso del tiempo, como se puede evidenciar en el contexto actual una forma de enseñanza arcaica sigue enmarcada, esto a pesar de los esfuerzos de las naciones por modificar la situación a través de nuevas estrategias pedagógicas, pues hay una fuerte consistencia por parte de los docentes y en general por las comunidades educativas a la falta de creatividad. Sin embargo, se han introducido elementos significativos para el tema educativo, estos elementos hacen parte de las tendencias de los países más desarrollados, las tecnologías de la información y la comunicación han permitido en la EF cambios importantes en su estructura, afectando a los países pobres, incluyendo algunos de Latinoamérica. Los directivos y docentes, han debido pensar en una nueva EF, una educación ligada a la formación de competencias, a la integralidad de los contenidos y habilidades para la vida, siendo necesario reformular el currículo de nuestra área.

En el presente estudio hace alusión al trabajo de Bart Crum (2017), quien comenta que en países de todo el mundo la EF escolar está navegando en aguas turbulentas y en peligro real de perder su posición en el currículo, esta situación se debe a la confusión y fracaso de la propia profesión, donde demasiados educadores físicos hablan de "enseñar EF" sin ninguna evidencia de la intención de producir aprendizaje. En consecuencia, nuestra profesión, en la realidad de nuestras escuelas públicas no logra el perfil de la actividad de enseñanza-aprendizaje relevante y por lo tanto está perdiendo apoyo político y social.

Por otra parte, también encontramos frases o expresiones que hacen referencia a contenidos de salud de manera implícita, a decir tres ámbitos de salud. Dentro del componente biológico de salud se encontraría como finalidad principal del área curricular desarrollar la competencia motriz, las habilidades motrices y el más importantes, el desarrollo de las actividades físicas. En la esfera de salud social, en- contramos el trabajo en equipo, colaboración, resolución de conflictos mediante el diálogo, desarrollo de la iniciativa individual, juego limpio y el respeto a las normas. Por último, dentro de la salud psicológica, aparece el desarrollo de actitudes como la valoración y el sentimiento acerca de sus propias limitaciones y posibilidades, reflexión, análisis, así como el disfrute de la práctica (Pastor-Vicedo, GilMadrona, Prieto-Ayuso y González-Víllora, 2015).

Es por esto que Zapata (2018), argumenta que cuando se habla de un diseño curricular para un área específica como lo es la $\mathrm{EF}$, se deben integrar otras disciplinas o ciencias que la complementen tales como lo son el deporte, la salud, la psicología y la biología; ya que estas áreas ayudan a fortalecer las acciones formativas correspondientes al ámbito de $\mathrm{EF}$.

Defender el carácter orgánico de un currículo permite, entre otras posibilidades, comprenderlo a partir de una visión de integralidad, es decir, en la congruencia entre teorías, políticas y prácticas. Las intencionalidades teóricas y políticas de una propuesta curricular y de las acciones educativas necesitan ser comprendidas como indisociables, pertenecientes a un continuo orgánico compuesto por fuerzas que consolidan y/o inestabilizan el todo, representando un movimiento contradictorio de influencias mutuas sin aislar los sentidos destacados, trabajando en el entendimiento de que apropiar es transformar, lo que se recibe en algo propio, produciendo sentidos y significados de manera inventiva (Tenório, Tavares, Oliveira, Neira y Júnior, 2017).

En lo que se refiere al referencial curricular, González (2016) describe que el papel del mismo debería cumplir en el proceso de planeación de la EF en las escuelas, afirma que el documento no fue pensado como un currículo común a ser desarrollado en toda la red de enseñanza pública; si no que se trata de un parámetro para auxiliar en la articulación entre los planes de estudios de la disciplina, los proyectos escolares específicos y la realidad local. En ese sentido, destaca el cuidado de seleccionar prácticas corporales que potencialicen la participación de todos los alumnos en actividades 
recreativas después de concluida la Educación Básica.

En la EF de algunos países de Iberoamérica, existen diversas problemáticas que los educadores físicos y otros responsables de impartirla en los diferentes niveles que requieren ser resueltas para promover intervenciones de prácticas pedagógicas que favorezcan a la comunidad educativa en la adquisición de aprendizajes, con los cuales puedan enfrentar los retos que la realidad les impone en la sociedad. Como concerniente de este estudio se verán plasmadas algunas citas muy enfocadas a las situaciones actuales de algunos países.

En vistas de proponer el deslizamiento de una EF a una Educación Corporal, Rodríguez (2018) propone que se debe recuperar críticamente la enseñanza por contenidos, suponer a la enseñanza como un proceso que se constituye en y por el lenguaje, incorporar el problema de la transmisión que involucra aquello que no puede ser representado a través del lenguaje, reconceptualizar la teoría curricular a partir de implicar al sujeto, reconocer al sujeto no como una representación sino como producto de un discurso, suponer al saber en su falta, situar a la práctica como modo de hacer, pensar y decir, e incorporar la noción de uso.

También se debe destacar que las pocas condiciones de las clases de EF, como, por ejemplo, carencia de materiales, cantidad excesiva de alumnos, entre otros factores, pueden no contribuir para que tal objetivo, de carácter estrictamente funcional, sea alcanzado. Además de los enunciados provenientes de las teorías curriculares psicomotora, desarrolladora y deportiva, hay que destacar también en los currículos examinados, enunciados relacionados a los presupuestos del currículo sano (da Silva y de Oliveira, 2018).

Actualmente se sigue trabajando para lograr que los currículos tengan cada vez más requisitos para una EF de Calidad, autores plantean que se hace necesario establecer, para resolver el reto que actualmente tiene la EF en Ecuador, entre otros aspectos, el desarrollar una metodología de trabajo articulada y en equilibrio con los nuevos escenarios educativos y sociales que se enfrentan en el país. Se precisa un repensar científico en cuanto a la relación que debe darse de manera indisoluble entre currículo y las alternativas didácticas metodológicas que se desarrollan actualmente en las clases de EF y su relación con el proceso de evaluación, como una alternativa para elevar el rendimiento motriz y educativo de los estudiantes y la calidad de las sesiones (HerreraMena, Tocto-Lobato, Lomas-Badillo y MazónMoreno et al., 2018).

En un estudio realizado por los autores Portillo-Torres, Hernández-Quesada, y QuirósQuirós (2016), sobre el proyecto curricular de Costa Rica, de acuerdo con la opinión de maestros y estudiantes presenta dificultades, se vuelve más teórica la sesión quitando tiempo para realizar actividades físicas, no tomando en cuenta el trabajo en clase y la asistencia, pues tanto estudiantes como docentes describen una práctica común, estudiantes que no asisten a clase y solo presentan el trabajo escrito. Además, el personal docente revisa los avances en clase, lo cual le quita aún más tiempo. Por lo que el estudiantado ve la clase como sedentaria.

Igualmente se ha podido constatar que algunos de los resultados de este estudio coinciden con un trabajo anterior, en relación a la consideración de la evolución de los intereses y opiniones curriculares del área de EF de padres y alumnos. La EF como la asignatura importante para su formación, contrasta con las otras asignaturas, pues en la actualidad se percibe tanto por alumnos como de padres, como poco valorada en relación a su contribución en la formación educativa, esto puede deberse a que uno de los centros de Educación Primaria, así como el Instituto de Educación Secundaria, se han convertido en instituciones bilingües, aumentando la valoración de la asignatura troncales (Gil-Madrona, Perona-Andres, PrietoAyuso y Saez-Sánchez, 2017).

Por otro lado, un currículum centrado en la corporeidad, como el mexicano, no promueve tanto el movimiento y la actividad, como otros programas con enfoques más diversos como el brasileño y español, o incluso, el argentino que ha adoptado los mismos enfoques que el 
mexicano. Esto parece contradictorio con las tendencias actuales de la $\mathrm{EF}$, a la que se le ha encomendado el fomento de la vida saludable, el movimiento y el deporte. Cabe señalar que existen algunos indicios de que no todos los educadores físicos mexicanos cumplen con los enfoques del programa vigente de EF centrado en la corporeidad (García y Del Basto 2017).

Por lo anterior expuesto se propone el siguiente objetivo: Analizar las problemáticas sobre el currículo actual de la Educación Física en Iberoamérica, a través de una revisión sistemática que nos brinde información transcendental para conocer la situación vigente.

\section{MÉTODO}

Como fuentes de información para la búsqueda de artículos, se recurrió a las siguientes bases de datos electrónicas: "Scielo", "Scopus", "Web of Science," "Redalyc" y Google académico. Utilizando los siguientes descriptores (en Portugués/Inglés/Español): "Curricular", Educación Física, "Iberoamérica" y con los criterios de inclusión siguientes, artículos de investigación teórica y/o empírica con resumen y textos completos disponibles para el análisis, entre los años 2013 y 2018. Eliminando los archivos duplicados, logrando un total de 1310 , de los cuales 1049 fueron no elegibles por términos del título, 226 por términos de resumen, 29 no elegibles por revisión de texto completo, quedando solo 6 estudios que cumplieron con los criterios inclusión establecidos, de los que se rescató el título, resumen, resultados y discusión para su análisis final.

Metodología de búsqueda propuesta por los autores: Ferreira, Urrutia y Alonso-Coello (2011), la cual se describe a continuación (Figura 1).

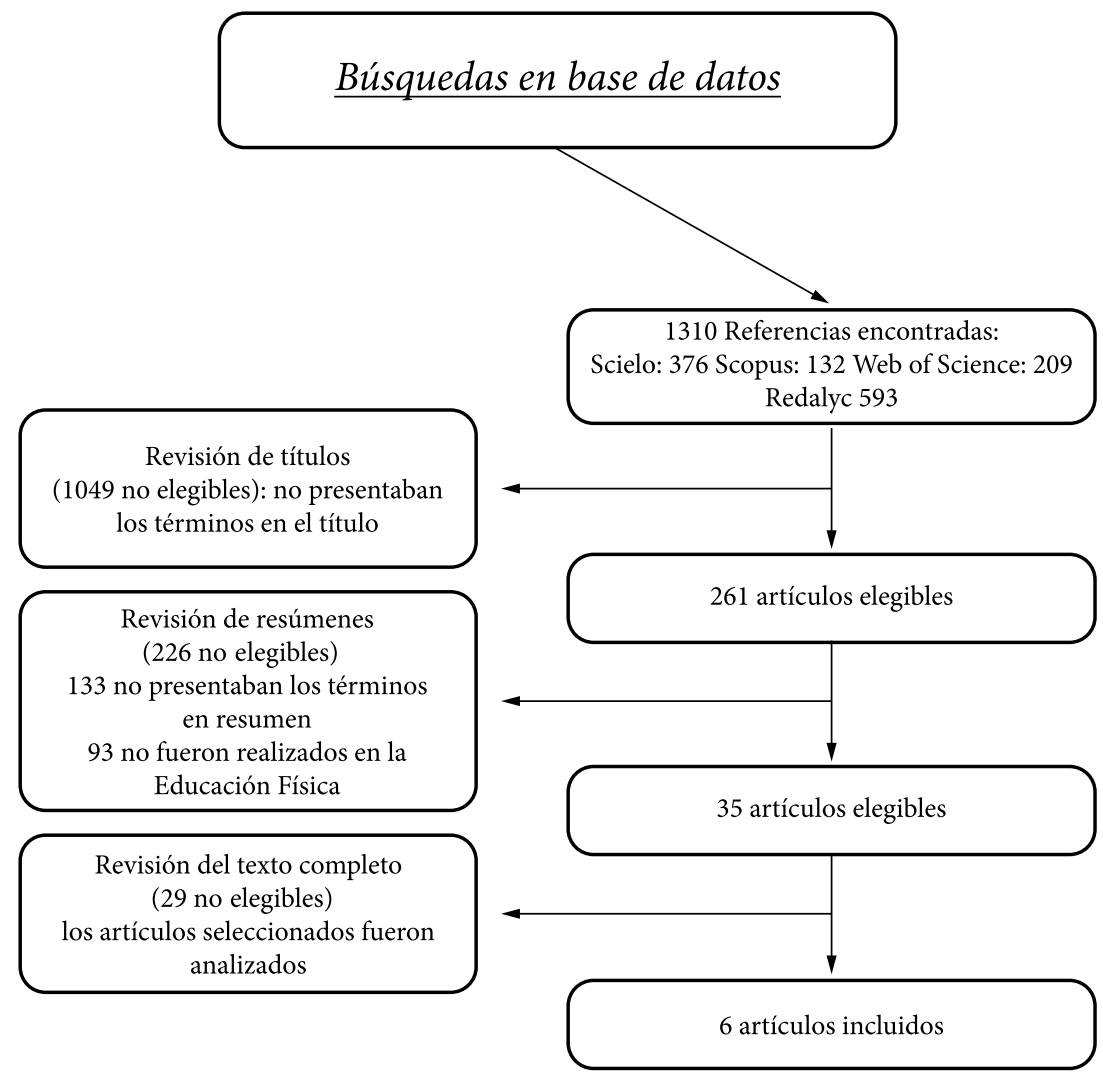

Figura 1. Diagrama para búsqueda en bases de datos. 


\section{RESULTADOS}

Como se puede apreciar los estudios por países, los cuales nos hablan sobre los currículos actuales de la EF en Iberoamérica, así como de los problemas curriculares tanto en la educación básica, primaria y secundaria, de países como: Brasil, España, México, Ecuador, Costa Rica y Argentina, las cuales están muy relacionados. Además de que los currículos están diseñados en términos de competencias, se puede observar que en los objetivos curriculares de algunos de dichos países se manifiestan los enfoques del currículo como los de corporeidad. Que a continuación se muestran puntualmente caso por caso a partir de los resultados obtenidos (Tabla 1).

Los autores Gil-Madrona et al. (2017) afirman, en sentido general, que la valoración de toda la comunidad educativa pasa por los diferentes componentes del currículum percatándose que puede ser responsabilidad del profesor y de su falta de interés por revertir la situación, de algunos factores que alientan la falta de importancia de la familia y del alumnado por la clase de EF. 


\section{Tabla 1}

Resultados de los Análisis curriculares actuales de los estudios de la EF en Iberoamérica.

\begin{tabular}{|c|c|c|}
\hline $\begin{array}{l}\text { Autor, Año y } \\
\text { País }\end{array}$ & Título & Objetivo \\
\hline $\begin{array}{l}\text { Gil-Madrona et } \\
\text { al. (2017) } \\
\text { España }\end{array}$ & $\begin{array}{l}\text { Evolución de los inte- } \\
\text { reses y opiniones cu- } \\
\text { rriculares del área de } \\
\text { Educación Física de } \\
\text { padres y alumnos. }\end{array}$ & $\begin{array}{l}\text { Conocer el interés de pa- } \\
\text { dres y alumnos por la EF, y } \\
\text { si dicho interés ha variado } \\
\text { en la última década. }\end{array}$ \\
\hline $\begin{array}{l}\text { Herrera-Mena } \\
\text { et al. }(2018) \\
\text { Ecuador }\end{array}$ & $\begin{array}{l}\text { Currículo, evaluación } \\
\text { y calidad en Educación } \\
\text { Física: retos y prospec- } \\
\text { tivas en el contexto } \\
\text { ecuatoriano. }\end{array}$ & $\begin{array}{l}\text { Reflexionar sobre la im- } \\
\text { portancia de relación en- } \\
\text { tre currículo, evaluación y } \\
\text { calidad. Y los retos y pros- } \\
\text { pectivas que se presentan } \\
\text { actualmente en la clase de } \\
\text { EF. }\end{array}$ \\
\hline $\begin{array}{l}\text { García et al. } \\
(2017) \\
\text { México }\end{array}$ & $\begin{array}{l}\text { Revisión Internacional } \\
\text { e Iberoamericana del } \\
\text { curriculum de Educa- } \\
\text { ción Física. El caso de } \\
\text { México. }\end{array}$ & $\begin{array}{l}\text { Identificar tendencias } \\
\text { curriculares de EF en el } \\
\text { medio internacional y } \\
\text { analizar cómo se han in- } \\
\text { corporado en los objetivos } \\
\text { del currículo de países } \\
\text { como Argentina, Brasil, } \\
\text { España y particularmente } \\
\text { en México. }\end{array}$ \\
\hline
\end{tabular}

\begin{tabular}{lll}
\hline da Silva y de & Estudio curricular & Analizar la producción \\
Oliveira (2018) & supervisado en la for- & de conocimiento sobre el \\
Brasil & mación de profesores & estudio curricular super- \\
& de educación física en & visada en la formación de \\
& Brasil: una revisión sis- & profesores de EF. \\
& temática.
\end{tabular}

1. Se sigue otorgando poca importancia a la EF como materia formativa.

2. Pero sigue siendo la asignatura que más interés y motivación despierta en el alumnado.

1. La necesidad de un repensar científico sobre la relación entre dichos elementos curriculares que se dan actualmente.

2. Lo que llevaría elevar la calidad de las clases de EF y por ende el rendimiento motriz y educativo de los estudiantes.

1. El currículum mexicano no ha incorporado las tendencias mundiales actuales, que dan prioridad al movimiento e iniciación deportiva.

2. Este hallazgo plantea serios retos a la EF mexicana.

3. Es posible presumir que debido a una de las principales orientaciones del programa, la corporeidad, además de las instalaciones y materiales con las que cuentan, no favorecen el seguimiento de dichas tendencias.

1. Se concluye que hubo aumento en la producción del conocimiento científico en la capacitación del docente de EF.

2. Pero se resalta sobre la importancia, en los últimos años, de fortalecer la producción con mayor calidad, evitando repetición o temas innecesarios.

Portillo-Torres Opinión de docentes Describir los aspectos más et al (2016) y estudiantes sobre las relevantes de las lecciones Costa Rica clases de Educación Física en secundaria. a partir del enfoque de la reforma curricular.
Rodríguez

(2018)

Argentina
Contenidos de la enseñanza de la Educación Física escolar. Discusiones en torno al currículo en Argentina.
Analizar el recorrido discursivo de la reforma en relación con las practicas escolares a las que estuvo destinado, a fin de recuperar críticamente los Contenidos Básicos Comunes (CBC) como expresión concreta de una pedagogía sostenida en la enseñanza de contenidos.
1. Tanto docentes como alumnos perciben que las clases de EF cambiaron de una manera favorable.

2. Pero también se encontró que no existe claridad conceptual del nuevo enfoque.

3. Situación que ha dado pie al ensayoerror y que no ha sido resuelta por la asesoría recibida.

1. Se encontró que la transición de una $\mathrm{EF}$ a una Educación Corporal tiene su sustento o correlato, la primera con la psicología del desarrollo y la segunda con el psicoanálisis.

2. Pero también, en la propia constitución de su objeto, en tanto la primera trata con conocimientos y la segunda con el saber.

3. Lograr un trabajo sobre sus acciones, sus senaciones, sus emociones, sus deseos, de una menera intelingente $y$ con un sentido social permite la integracion de l corporeidad como bas de aprendizaje en la EF. 
En los contextos sociales se viven nuevos retos que inciden en la comunidad educativa, en este sentido Herrera-Mena et al. (2018) plantean reflexionar o meditar sobre la importancia de los lineamientos curriculares cómo pensar, hacer y enseñar una EF que dé respuesta a los factores que intervienen en su realización, creando una estrecha relación entre los modelos curriculares que aporten a una calidad de vida en torno a las prácticas de actividad física en las escuelas.

Por otro lado, el estudio de los objetivos curriculares nos brinda la oportunidad de profundizar en dichos contextos sociales de cada lugar, por su parte García y Del Basto (2017); Rodríguez (2018) analizaron, haciendo comparaciones de programas y sus principales orientaciones con la corporeidad, que ellos consideran no favorecen el seguimiento de las nuevas tendencias. La problemática de estos hallazgos se ve reflejadas en las mayas curriculares de la EF, pudiendo tener diferentes cambios para alcanzar transformaciones en los diseños curriculares, creando nuevos fundamentos legales, nuevas concepciones psicopedagógicas y procesos didácticos, lo que implicaría una nueva forma de administración curricular y unas condiciones de ejecución que respondan a los nuevos contextos.

De acuerdo, con da Silva y de Oliveira (2018), quienes analizan sobre el estudio curricular supervisado en la formación de profesores de EF, revelan varios aspectos importantes en el conocimiento y la trayectoria epistemológica en esta área, con el fin de facilitar la comprensión de la situación actual, así como identificación de trayectorias y direcciones a explorarse más adelante, ellos apoyan la idea de considerar los aspectos analizados con el fin de evitar la duplicación información o investigación innecesaria y sobre todo, para llenar las vacíos que se presentan actualmente en la rama de la educación física y preferentemente en los educadores físicos.
Por su parte, Portillo-Torres et al. (2016) quienes describen las percepciones positivas de los estudiantes de EF y sus profesores, muestran cambios sustanciales que se pudieron ver en el proceso investigativo. No obstante, el enfoque de la reforma curricular a la que deriva para la preparación profesional de los profesores implica una particular forma de relacionar teoría y práctica, que aparecen fragmentadas y disociadas en dicho currículo, por lo cual los espacios curriculares de prácticas están muy diversificadas y sin claridad al nuevo enfoque, que solo es posible con una planificación adecuada, no necesariamente a prueba y error, así como con buen uso del material disponible, o con las herramientas didácticas necesarias para la obtención de buenos resultados.

A criterio final de los autores del presente trabajo, haciendo un esfuerzo de síntesis, que el currículo continuará siendo lo medular de lo intelectual y organizativo de los procesos educativos en la EF, pero entran en una complejidad de factores como las concepciones didácticas curriculares, teniendo como constancia la falta de comprensión teórico-práctico, de forma conceptual y apropiación de los modelos educativos, además atados a la necesidad de procesos adecuados de formación docente de los educadores físicos para el proceso de transformación de la enseñanza en las clase de $\mathrm{EF}$, así como el apoyo social de parte de los padres, asesores y maestros. Finalmente, los educadores físicos se mantienen en un proceso de cambio de concepciones y prácticas que va mucho más allá de un simple aprendizaje, para poder lograr cambio del currículo se requiere una secuencia sistemática de trabajo, que trate todos los aspectos, las condiciones para el trabajo productivo, así como de profesionales capacitados de una gran preparación, nuevas destrezas, nuevas perspectivas cognoscitivas, nuevos modos de pensamiento y con cambio de actitudes de la personas hacia lo importante, las percepciones acerca de las funciones, los propósitos y la motivación. 


\section{CONCLUSIONES}

- No es ajeno a nuestro entorno la falta reconocimiento social que ostenta la $\mathrm{EF}$, así mismo, que es bien recibida en los patios escolares; esto no necesariamente sobre la base de la calidad de la labor profesional de nuestros colegas, sino más bien a esa necesidad de movimiento innata en la niñez.

- Por lo que se vuelve necesario que dicha práctica profesional se ancle sobre el quehacer pedagógico-científico, si queremos aspirar a esa EF de Calidad tan mencionada en la actualidad por expertos y autoridades internacionales.

- Se denuncia esa poca claridad conceptual, ese ensayo error; esa falta de sustento científico, esa incongruencia que ha existido en los distintos intentos por llevar a cabo diversas acepciones curriculares, así mismo se ponen de manifiesto los problemas para su implementación desde la integralidad.

- Podemos sugerir que es de suma importancia que el currículo de la EF debe asumirse como un proyecto educacional flexible, adaptable a los cambios educativo actuales, con un enfoque integral que abarque no solo los aprendizajes obtenidos en el currículo de nuestra profesión, sino también los aprendizajes que se adquieren en otros ámbitos del propio sistema educativo, con los cuales ofrecer respuestas efectivas a los problemas perceptibles generados por necesidades no cubiertas. 


\section{REFERENCIAS BIBLIOGRÁFICAS}

Crum, B. (2017). How to win the battle for survival as a school subject? Reflections on justification, objectives, methods and organization of $\mathrm{PE}$ in schools of the 21st century. Retos: nuevas tendencias en educación física, deporte y recreación, 31, 238-244.

da Silva Júnior, A. P., \& de Oliveira, A. A. B. (2018). Estudio curricular supervisado en la formación de profesores de educación física en Brasil: una revisión sistemática. Movimento, 24(1), 77-92.

Ferreira, I., Urrútia, G., Alonso-Coello, P. (2011). Revisiones sistemáticas y metaanálisis: bases conceptuales e interpretación. Revista Española de Cardiología, 64(8), 688-696.

García, J. M., \& Del Basto, L. M. (2017). Revisión Internacional e Iberoamericana del Currículum de Educación Física. El Caso de México. REICE. Revista Iberoamericana sobre Calidad, Eficacia y Cambio en Educación, 15(1), 71-85.

González, F. (2016). Desafíos para la educación física escolar brasileña: una propuesta de currículum. Retos: nuevas Tendencias en Educación Física, Deporte y Recreación, 29, 188-194.

Gil-Madrona, P., Perona-Andres, J., PrietoAyuso, A., \& Saez-Sánchez, M. (2017). Evolución de los intereses y opiniones curriculares del área de Educación Física de padres y alumnos. Movimento, 23(3), 1065-1078.

Herrera-Mena, V. H., Tocto-Lobato, J. G., Lomas-Badillo P.L. \& Mazón-Moreno O. D. (2018). Currículo, evaluación y calidad en Educación Física: retos y prospectivas en el contexto ecuatoriano. Olimpia: Publicación científica de la Facultad de Cultura Física de la Universidad de Granma, 15(47), 47-56.
Portillo-Torres, M. C., Hernández-Quesada, Ó., \& Quirós-Quirós, H. (2016). Opinión de docentes y estudiantes sobre las clases de educación física en secundaria. Revista Electrónica Educare, 20(2).

Pastor-Vicedo, J. C., Gil-Madrona, P., Prieto-Ayuso, A., \& González-Víllora, S. (2015). Los contenidos de salud en el área de educación física: Análisis del currículum vigente. Retos: nuevas Tendencias en Educación Física, Deporte y Recreación, (28).

Rodríguez, N. B. (2018). Contenidos de la enseñanza de la educación física escolar. Discusiones en torno al currículo en Argentina. Revista Tempos e Espaços em Educação, 11(25), 21-32.

Tenório, K. M. R., Tavares, M., Oliveira, R., Neira, M., \& Júnior, M. S. (2017). Apropriações e produções curriculares de professores de educação física. Movimento, 23(4), 1177.

Zapata, D. (2018). Una propuesta curricular en educación física para los estudiantes de la Escuela Universidad Nacional sede Medellín, teniendo en cuenta algunos lineamientos de formación para la promoción de la salud en el 2017. (Trabajo de grado Licenciatura en Educación Física y Deportes). Universidad de San Buenaventura Colombia, Facultad de Educación, Medellín.

\section{Dirección para correspondencia}

Dr. Gabriel Gastélum

Facultad de Ciencias de la Cultura Física

Universidad Autónoma de Chihuahua

México

Contacto:

gastelum@uach.mx

Recibido: 06/06/2018

Aceptado: 28/06/2018 